A Unified Approach to B-Spline Recursions and Knot Insertion, with Application to New Recursion Formulas

Guido Walz

Nr. 153

April 1993 


\title{
A Unified Approach to B-Spline Recursions and Knot Insertion, with Application to New Recursion Formulas
}

\author{
Guido Walz
}

\begin{abstract}
We present a unified approach to and a generalization of almost all known recursion schemes concerning B-spline functions. This includes formulas for the computation of a B-spline's values, its derivatives (ordinary and partial), and for a knot insertion method for B-spline curves. Furthermore, our generalization allows us to derive interesting new relations for these purposes.
\end{abstract}

Keywords. B-Splines, Recursion Schemes, Knot Insertion, Contour Integral.

Abbreviated Title. B-Spline Recursion and Knot Insertion 


\section{Preliminaries}

B-spline functions and curves are nowadays important tools in many fields of mathematics and engineering, and in the meantime there exist a lot of recursion formulas for the computation of a B-spline's values (e.g. [deBoor 1972], [Cox 1972]), its derivative (e.g. [deBoor 1972], Meinardus [1984]), its partial derivative w.r.t. the knots (e.g. [Schumaker 1982], [Meinardus\&Walz 1993]) and for knot insertion (e.g. [Boehm 1980]). A compendium of these relations can be found in many textbooks, such as [deBoor 1978], [Farin 1988], [Hoschek\&Lasser 1989], [Nürnberger 1989], [Schumaker 1981].

In this paper we would like to present a unified approach to these formulas; we will do this by proving generalized relations for each of the above-mentioned situations: Bspline value recursions in Section 2, formulas for a B-spline's derivatives in Section 3, and knot insertion in Section 4. Our generalizations do not only cover almost all formulas mentioned in the first paragraph as special cases, but they allow also to derive quite easily some interesting new relations.

The approach we are going to present is in all cases based on the very nice contour integral representation of B-splines (1.2), due to G. Meinardus [Meinardus 1974].

\section{Introduction}

Let us be given natural numbers $m$ and $k$ with $m \geq k \geq 1$, and a set of knots $x_{\nu} \in \mathbb{R}, \nu=0, \ldots, k$, satisfying

$$
x_{0}<x_{1}<\cdots<x_{k} .
$$

To each knot $x_{\nu}$ there is associated a natural number $r_{\nu}$, called the multiplicity of $x_{\nu}$ such that

$$
r_{0}+r_{1}+\cdots+r_{k}=m+1 \text {. }
$$

We call a real function $f$ a (polynomial) $B$-spline of order $m$, belonging to the set of knots $\left\{x_{\nu}\right\}$ with multiplicities $r_{\nu^{\prime}}, \nu=0, \ldots, k$, if it possesses the following properties:

1. It is

$$
f(x)=0 \quad \text { for all } x<x_{0} \text { and for all } x>x_{k} ;
$$

2. The restriction of $f$ to the subinterval $\left[x_{\nu}, x_{\nu+1}\right), \nu=0, \ldots, k-2$, and to $\left[x_{k-1}, x_{k}\right]$ belongs to the space $\Pi_{m-1}$ of polynomials of degree at most $m-1$.

3. If $r_{\nu}<m$, it is

$$
f^{\prime} \in C^{m-1-r_{\nu}}\left(U\left(x_{\nu}\right)\right)
$$


for a suitable neighborhood $U\left(x_{\nu}\right)$ of the knot $x_{\nu}, \nu=0,1, \ldots, k$,

4. We have

$$
\int_{-\infty}^{+\infty} f(x)=1
$$

It is well-known that there exists one and only one such function $f$. It is called $B$-spline of order $m$ and denoted by

$$
B_{m}\left(x \mid \begin{array}{llll}
x_{0} & \cdots & \cdots & x_{k} \\
r_{0} & \cdots & \cdots & r_{k}
\end{array}\right)
$$

In the case of simple knots, we will also use the notation

$$
B_{m}^{\mathbf{1}}(x):=B_{m}\left(x \mid \begin{array}{cccc}
x_{0} & \cdots & \cdots & x_{m} \\
1 & \cdots & \cdots & 1
\end{array}\right)
$$

and

$$
B_{m-1}^{1}\left(x ; \# x_{\nu}\right):=B_{m-1}\left(x \mid \begin{array}{ccccccc}
x_{0} & \cdots & x_{\nu-1} & x_{\nu} & x_{\nu+1} & \cdots & x_{m}^{\prime} \\
1 & \cdots & 1 & 0 & 1 & \cdots & 1
\end{array}\right)
$$

where of course a zero multiplicity indicates that the corresponding knot is no longer an active one for this $B$-spline.

The basic theory of these functions is well-developed and can be found in any textbook on spline functions ([deBoor 1978], [Schumaker 1981], [Nürnberger 1989]) or CAGD ([Farin 1988], [Hoschek\&Lasser 1989]), where sometimes other approaches and definitions ' are taken, but which of course lead all to the same functions.

Furthermore it should be noted that there exist in the literature also other normalizations than the one given under \#4. Sometimes the integral is set equal to $1 / m$, the resulting B-spline being denoted by $Q_{m}(x)$, and in other approaches to $\left(x_{k}-x_{11}\right) / m$, which yields the normalized B-splines $N_{m i}(x)$. We stick to the normalization $\int B_{m}=1$, which is historically the correct one; however, since all these functions are related by

$$
B_{m}=m \cdot Q_{m}=\frac{m}{x_{k}-x_{0}} \cdot N_{m}
$$

all of the following results carry over to the $Q_{m}$ 's and $N_{m}$ 's by a simple multiplication with constants.

Our fundamental tool will be the following contour integral representation of a Bspline, which was proved by G. Meinardus in [Meinardus 1974] and further investigated in several publications, see [Meinardus\&Walz 1993]. 
Theorem 1.1 [Meinardus 1974]: Let, for $x \in \mathbb{R}, C=C_{x}$ denote a simply closed and rectifiable curve in the complex plane, such that all the knots $x_{\nu}, \nu \in\{0, \ldots, k\}$, with $x<x_{\nu}$ and no others lie in the interior of that curve.

Then, carrying out the integration in the positive sense, we have

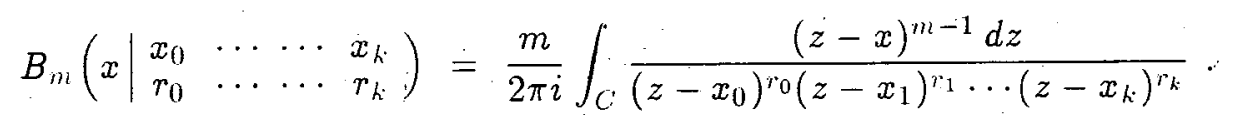

The representation (1.2) can serve as a theoretical tool as well as a practical instrument for the computation of a B-spline, via the residue theorem (see [Meinardus\&Walz 1993]).

\section{B-Spline Value Recursions}

We begin this section by proving the following general B-spline recursion formula:

Theorem 2.1: With some natural number $n, n \leq r_{\nu}$ for $\nu=0, \ldots, k$, let there be given real functions $\lambda_{\nu}, \nu=0, \ldots, k$, satisfying

$$
\sum_{\nu=0}^{k} \lambda_{\nu}(x) \cdot x_{\nu}^{j}=x^{j}, j=0, \ldots, n .
$$

Then the following recursion formula holds:

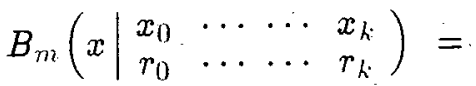

$$
\begin{aligned}
& =\frac{m}{m-n} \cdot \sum_{\nu=0}^{k} \lambda_{\nu}(x) \cdot B_{m-n}\left(x \mid \begin{array}{ccccccc}
x_{0} & \cdots & x_{\nu-1} & x_{\nu} & x_{\nu+1} & \cdots & x_{k} \\
r_{0} & \cdots & r_{\nu-1} & r_{\nu}-n & r_{\nu+1} & \cdots & r_{k}
\end{array}\right) \text {. }
\end{aligned}
$$

Remark. In a recently published thesis by M. Neamtu [Neamtu 1991] one can find two formulae which turn out to be special cases of (2.2) (resp. (3.2) below) for $n=1$ and the case of simple knots. The author traces those formulae back to [Dahmen 1980] and [Micchelli 1979], who found independently a corresponding relation for multivariate simplex splines. However, although more general, the following proof is surely more elementary than the one in the above-mentioned publication.

Proof of Theorem 2.1. Let us for the moment use the abbreviations

$$
\omega_{\nu, n}(z):=\frac{\prod_{j=0}^{k}\left(z-x_{j}\right)^{r_{j}}}{\left(z-x_{\nu}\right)^{n}}, \quad \nu=0, \ldots, k
$$

and

$$
B_{m-i l}^{\nu}(x):=B_{m-n}\left(x \mid \begin{array}{ccccccc}
x_{0} & \cdots & x_{\nu-1} & x_{\nu} & x_{\nu+1} & \cdots & x_{k} \\
r_{0} & \cdots & r_{\nu-1} & r_{\nu}-n & r_{\nu+1} & \cdots & r_{k}
\end{array}\right)
$$


Then, according to (1.2), we have

$$
B_{m-n}^{\nu}(x)=\frac{m-n}{2 \pi i} \int_{C} \frac{(\dot{z}-x)^{m-n-1} d z}{\omega_{\nu, n}(z)}
$$

and therefore

$$
\begin{aligned}
\frac{m}{m-n} \cdot \sum_{\nu=0}^{k} \lambda_{\nu}(\dot{x}) \cdot B_{m-n}^{\nu}(x) & =\frac{m}{2 \pi i} \int_{C} \frac{\sum_{\nu=11}^{k} \lambda_{\nu}(x)\left(z-x_{\nu}\right)^{n} \cdot(z-x)^{m-n-1} d z}{\left(z-x_{0}\right)^{r_{0}}\left(z-x_{1}\right)^{r_{1}} \cdots\left(z-x_{k}\right)^{r_{k}}} \\
& =\frac{m}{2 \pi i} \int_{C} \frac{(z-x)^{n} \cdot(z-x)^{m-n-1} d z}{\left(z-x_{0}\right)^{r_{0}}\left(z-x_{1}\right)^{r_{1}} \cdots\left(z-x_{k}\right)^{r_{k}}}
\end{aligned}
$$

by means of (2.1) and the Binomial Theorem. This proves (2.2).

Before analyzing this general formula in more detail, we note that it covers as a very special case the standard recursion formula.

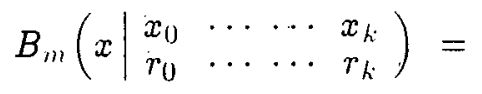

$$
\begin{aligned}
& =\frac{m}{m-1} \cdot\left(\frac{x-x_{0}}{x_{k}-x_{0}} B_{m-1}\left(x \mid \begin{array}{llll}
x_{0} & \cdots & x_{k-1} & x_{k} \\
r_{0} & \cdots & r_{k-1} & r_{k}-1
\end{array}\right)\right. \\
& \left.+\frac{x_{k}-x}{x_{k}-x_{0}} B_{m-1}\left(x \mid \begin{array}{cccc}
x_{0} & x_{1} & \cdots & x_{k} \\
r_{0}-1 & r_{1} & \cdots & r_{k}
\end{array}\right)\right),
\end{aligned}
$$

which is due to [deBoor 1972] and [Cox 1972].

If we assume to have simple knots and fix some value $\xi \in \mathbb{R}$, the assertion of Theorem 2.1 can also be interpreted as follows:

Corollary 2.2: If the point $\xi$ is written as the weighted sum of the knots $x_{\nu}$,

$$
\xi=\sum_{\nu=0}^{m} \lambda_{\nu} x_{\nu} \text { with } \sum_{\nu=0}^{m} \lambda_{\nu}=1,
$$

then the value of the $B$-spline $B_{m}^{1}$ at $\xi$ is the weighted sum of the B-spline, values $B_{m-1}^{1}\left(\xi ; \# x_{\nu}\right)$ with the same coefficients $\lambda_{\nu}$ :

$$
B_{m}^{1}(\xi)=\frac{m_{1}}{m-1} \cdot \sum_{\nu=0}^{m} \lambda_{\nu} B_{m-1}^{1}\left(\xi ; \# x_{\nu}\right) .
$$

As an example, let $\xi_{0}$ denote the Greville abscissa

$$
\xi_{0}=\frac{x_{1}+\cdots+x_{m-1}}{m-1}
$$

Then

$$
B_{m}^{1}\left(\xi_{0}\right)=\frac{m}{(m-1)^{2}} \cdot \sum_{\nu=0}^{m} B_{m-1}^{1}\left(\xi_{0} ; \# x_{\nu}\right)
$$


Proof. Set $\lambda_{0}=\lambda_{m}=0$ and $\lambda_{\mu}=1 /(m-1)$ for $\nu=1, \ldots, m-1$, and apply Corollary 2.2 .

We now come back to the general case and ask for the existence of functions $\lambda_{\nu}$, which satisfy (2.1) and are as simple as possible. The answer is given in

Theorem 2.3: Adopt the notation of Theorem 2.1, and assume furthermore that $n \leq k$.

Then the following statements hold:

a) There exist $k+1$ polynomials $\lambda_{0}, \ldots, \lambda_{k} \in \Pi_{n}$, such that (2.1) is satisfied.

b) For each choice of $n+1$ distinct indices $\left\{\nu_{11}, \ldots, \nu_{n}\right\} \subset\{0, \ldots, k\}$, there exist $n+1$ uniquely determined polynomials $\lambda_{\nu^{\prime \prime}}, \ldots, \lambda_{\nu^{\prime \prime}} \in \Pi_{n}$, such that

$$
\sum_{\underline{k}=0}^{n} \lambda_{\nu_{\mathrm{g}}}(x) \cdot x_{\nu_{\mathrm{s}^{\prime}}}^{j}=x^{j}, j=0, \ldots, n
$$

(i.e. (2.1) is satisfied under the additional condition that

$$
\left.\lambda_{\nu} \equiv 0 \quad \text { for } \nu \in\{0, \ldots, k\} \backslash\left\{\nu_{0}, \ldots, \nu_{n}\right\}\right) .
$$

Proof. We only have to prove statement $\mathrm{b}$ ). To do this, we must show that there are coefficients $\alpha_{\nu_{s}, \mu}, \mu, \varrho=0, \ldots, n$, such that (2.7) holds with

$$
\lambda_{\mu_{0}}(x)=\sum_{\mu=11}^{n} \alpha_{\mu_{0}, \mu} x^{\mu}
$$

This means that, for $j=0, \ldots, n$, the polynomial identity

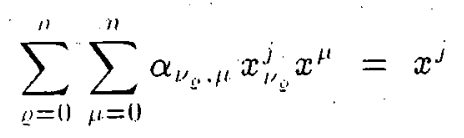

must hold. Comparing the coefficients of $x^{i}$ on both sides of (2.8) leads us to the $n+1$ systems of linear equations

$$
\sum_{\varrho=0}^{n} \alpha_{\nu^{\prime}, \mu} x_{\nu^{\prime},}^{j}= \begin{cases}1, & \text { if } j=\mu, \\ 0, & \text { if } j \in\{0, \ldots, n\}, j \neq \mu\end{cases}
$$

for $\mu=0, \ldots, n$. The matrix of these systems is a Vandermondian, hence regular, and so the existence and uniqueness of the coefficients $\alpha_{\mu_{y}^{\prime}, \mu}$ follows. 
Example. Take $n=1$, and set, for simplicity of the exposition, $\nu_{01}:=t$, and $\nu_{1}:=s$. Then the systems (2.9) have the solutions

$$
\begin{aligned}
& \alpha_{t 0}=\frac{x_{s}}{x_{s}-x_{t}}, \quad \alpha_{s 0}=\frac{-x_{t}}{x_{s}-x_{t}} \\
& \alpha_{t 1}=\frac{-1}{x_{s}-x_{t}}, \quad \alpha_{s 1}=\frac{1}{x_{s}-x_{t}}
\end{aligned}
$$

and we obtain, inserting this into (2.2), the recursion formula

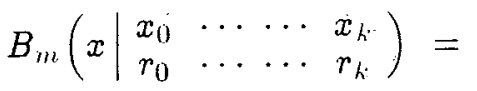

$$
\begin{aligned}
& =\frac{m}{m-1} \cdot\left(\frac{x_{s}-x}{x_{s}-x_{t}} \cdot B_{m-1}\left(x \mid \begin{array}{ccccccc}
x_{0} & \cdots & x_{t-1} & x_{t} & x_{t+1} & \cdots & x_{k} \\
r_{0} & \cdots & r_{t-1} & r_{t}-1 & r_{t+1} & \cdots & r_{k}
\end{array}\right)\right. \\
& \left.\left.+\frac{x-x_{t}}{x_{s}-x_{t}} \cdot B_{m-1}\left(x \mid \begin{array}{ccccccc}
x_{0} & \cdots & x_{s-1} & x_{s} & x_{s+1} & \cdots & x_{k} \\
r_{01} & \cdots & r_{s-1} & r_{s-1} & r_{s+1} & \cdots & r_{k}
\end{array}\right)\right) .\right\}
\end{aligned}
$$

Specializing once more to the case $t=0$ and $s=k$, we recover again the classical recursion formula (2.4).

Remark. The deBoor algorithm for the iterative computation resp. construction of a Bspline curve is surely one of the fundamental and most widespread algorithms in CAGD. Since it can be viewed as the B-spline-curve analogue to the classical recursion formula for the B-splines itself (using which it is also usually proved, see e.g. [Hoschek\&Lasser 1989]), it is clear that it would also be possible to formulate something like a "generalized deBooralgorithm", just using (2.1) instead of (2.4).

We close this section with a result of convolution type, connecting the B-splines with the Bernstein (basis) polynomials, defined for $k \in \mathbb{N}$ by

$$
P_{\nu}^{k}(x):=\left(\begin{array}{l}
k \\
\nu
\end{array}\right) x^{\nu}(1-x)^{k-\nu}, \nu=0, \ldots, k .
$$

(We note in passing that, since $P_{b}^{k}$ is also a B-spline, it possesses a contour integral representation of the type (1.2); but we will make no explicit use of this fact here). It is well-known that the Bernstein polynomials have linear precision w.r.t. the points $t_{\nu}:=\nu / k, \nu=0, \ldots, k$, i.e.

$$
\sum_{\nu=0}^{k} P_{\nu}^{k}(x)=1 \text { and } \sum_{\nu=0}^{k} t_{\nu} P_{\nu}^{k}(x)=x \text { for all } x .
$$

Using Theorem 2.1, we therefore have proved: 
Theorem 2.4: Consider the B-spline $B_{m}$ corresponding to the equidistant knots $t_{\nu}$, each of which is endowed with the multiplicity $r_{\nu}, \nu=0, \ldots, k$ (i.e. $m:=r_{0}+\cdots+$ $\left.r_{k}-1\right)$. Then the following recursion formula holds:

$$
\begin{aligned}
& B_{m}\left(x \mid \begin{array}{cccc}
t_{0} & \cdots & \cdots & t_{k} \\
r_{0} & \cdots & \cdots & r_{k}
\end{array}\right)= \\
& \quad=\frac{m}{m-1} \cdot \sum_{\nu=0}^{k} P_{\nu}^{k}(x) \cdot B_{m-1}\left(x \mid \begin{array}{ccccccc}
t_{0} & \cdots & t_{\nu-1} & t_{\nu} & t_{\nu+1} & \cdots & t_{k} \\
r_{0} & \cdots & r_{\nu-1} & r_{\nu}-1 & r_{\nu+1} & \cdots & r_{k}
\end{array}\right) .
\end{aligned}
$$

In particular; in the case of simple knots we have

$$
B_{m}^{1}(x)=\frac{m}{m-1} \cdot \sum_{\nu=0}^{m}\left(\begin{array}{c}
m \\
\nu
\end{array}\right) x^{\nu}(1-x)^{m i-\nu} \cdot B_{m-1}^{1}\left(x ; \# t_{\nu}\right)
$$

By a simple shift of variable it becomes clear that the results of Theorem 2.4 also hold for any B-spline with equally spaced knots.

\section{Derivatives and Partial Derivatives of B-Splines}

With the same approach as in the previous section, it is possible to find general recursion formulas for the derivatives of a B-spline; actually this is the case for the ordinary derivatives (i.e. with respect to the variable $x$ ). up to a certain order, as well as for the partial derivatives with respect to the knots $x_{1}$. Throughout this section we assume $m \geq 2$.

Theorem 3.1: With some natural number $n, n \leq r_{\nu}$ for $\nu=0, \ldots, k$, and $n<m$, let there be given real numbers $\lambda_{\nu}, \nu=0, \ldots, k$, satisfying

$$
\sum_{\nu=0}^{k} \lambda_{\nu} \cdot x_{\nu}^{j}= \begin{cases}0, & j=0, \ldots, n-1 \\ 1, & j=n\end{cases}
$$

Then the following recursion formula holds:

$$
\begin{aligned}
\frac{d^{n}}{d x^{n}} & B_{m}\left(x \mid \begin{array}{cccc}
x_{0} & \cdots & \cdots & x_{k} \\
r_{0} & \cdots & \cdots & r_{k}
\end{array}\right)= \\
\quad= & \frac{m !}{(m-n) !} \cdot \sum_{\nu=1}^{k} \lambda_{t^{\prime}} \cdot B_{m-n}\left(x \mid \begin{array}{lllllll}
x_{0} & \cdots & x_{\nu-1} & x_{\nu} & x_{\nu+1} & \cdots & x_{k} \\
r_{0} & \cdots & r_{\nu-1} & r_{\nu}-n & r_{\nu+1} & \cdots & r_{k}
\end{array}\right) .
\end{aligned}
$$


Proof. The proof is in analogy to that of Theorem 2.1, using the fact that

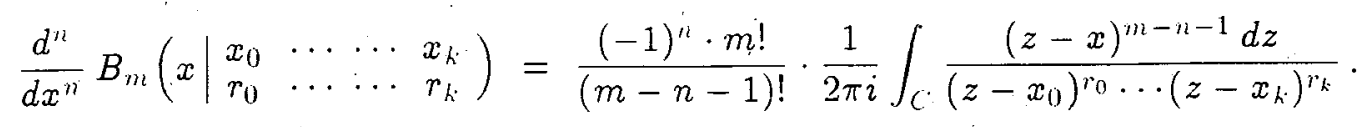

Equation (3.3) can be easily deduced from (1.2), see also [Meinardus\&Walz 1993].

It should be emphasized that here - in contrast to the recursions of the previous section - the $\lambda_{\nu}$ 's do not depend on $x$. Moreover, we can prove the following lemma, which gives us one possible choice of these numbers explicitly.

Lemma 3.2: Adopt the notation of Theorem 3.1, and assume in addition that $n \leq k$. Furthermore choose any subset of $n+1$ distinct indices $\left\{\nu_{0}, \ldots, \nu_{n}\right\}$ from $\{0, \ldots, k\}$. Then the real numbers $\lambda_{\nu}, \nu=0, \ldots, k$, defined by

$$
\begin{aligned}
\lambda_{\nu} & :=0 \text { for } \nu \in\{0, \ldots, k\} \backslash\left\{\nu_{0}, \ldots, \nu_{n}\right\}, \\
\lambda_{\nu_{g}} & :=\frac{1}{\prod_{\sigma=0 . \sigma \neq \varrho}^{n}\left(x_{\nu_{g}}-\dot{x}_{\nu_{\sigma}}\right)} \text { for } \varrho=0, \ldots, n
\end{aligned}
$$

satisfy relation (3.1).

Proof. We only have to apply Cramer's rule to the reduced system

$$
\sum_{\varrho=0}^{n} \lambda_{\nu_{e}} \cdot x_{\nu_{\mathrm{g}}}^{j}= \begin{cases}0, & j=0, \ldots, n-1, \\ 1, & j=n\end{cases}
$$

which yields the solutions

$$
\begin{aligned}
& \lambda_{\nu_{s}}=\frac{(-1)^{n+\varrho} \cdot \prod_{\substack{0 \leq \sigma<\tau \leq n \\
\sigma, r \neq q}}\left(x_{\nu_{r}}-x_{\nu_{\sigma}}\right)}{\prod_{0 \leq \sigma<\tau \leq n}\left(x_{\nu_{\tau}}-x_{\nu_{\sigma}}\right)} \\
& =\frac{(-1)^{n+e}}{\prod_{\substack{0 \leq \sigma \leq n \\
\sigma \neq \varrho}}\left(x_{\mu_{\rho}}-x_{\nu_{\sigma}}\right) \cdot(-1)^{n-e}} \\
& =\frac{1}{\prod_{\substack{\sigma=0 \\
\sigma \neq \varrho}}^{n}\left(x_{\nu_{\varrho}}-x_{\nu_{\sigma}}\right)} \quad \text { for } \varrho=0, \ldots, n \text {. }
\end{aligned}
$$

A closer look onto the system of equations (3.5) and its solutions (3.4) leads us to the following rather funny relation between the $n$-th derivative of a $\mathrm{B}$-spline and the $n-$ th order divided difference of certain other B-spline values: 
Theorem 3.3: Choose, as in Lemma 3.2, any $n+1$ distinct indices $\left\{\nu_{0}, \ldots, \nu_{n}\right\}$, and set for simplicity

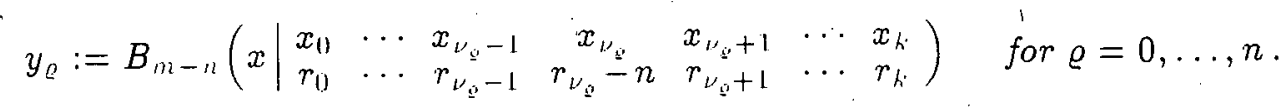

Then

$$
\frac{d^{n}}{d x^{n}} B_{m}\left(x \mid \begin{array}{cccc}
x_{1} & \cdots & \cdots & x_{k} \\
r_{0} & \cdots & \cdots & r_{k}
\end{array}\right)=\frac{m !}{(m-n) !} \cdot \Delta\left(x_{\iota^{\prime}}, \ldots, x_{\nu_{u}} ; y\right)
$$

where $\Delta$ denotes the usual divided difference operator, here applied to the vector $y=\left(y_{0}, \ldots, y_{n}\right)$.

Proof. It is well-known (see any textbook on Numerical Analysis) that the divided difference $\Delta$ of some function $\varphi$ can be written as a linear functional of the form

$$
\Delta\left(x_{\nu_{0}}, \ldots, x_{\nu_{n}} ; \varphi\right)=\sum_{\varrho=0}^{n} \lambda_{\nu_{\varphi}} \varphi\left(x_{\nu_{\underline{\varphi}}}\right)
$$

with the $\lambda_{\nu_{0}}$ 's as given in (3.4). Now the assertion follows from (3.2).

Example. We choose again $n=1$ and set $\nu_{0}:=s$ and $\nu_{1}:=t$. Then

$$
\lambda_{s}=\frac{1}{x_{s}-x_{t}} \quad \text { and } \quad \lambda_{t}=\frac{1}{x_{t}-x_{s}}
$$

which gives us the following recursion formula for the first derivative:

$$
\left.\begin{array}{r}
\frac{d}{d x} B_{m}\left(x \mid \begin{array}{rrrr}
x_{0} & \cdots & \cdots & x_{k} \\
r_{0} & \cdots & \cdots & r_{k}
\end{array}\right)= \\
=\frac{m}{x_{s}-x_{t}} \cdot\left(B_{m-1}\left(x \mid \begin{array}{lllllll}
x_{0} & \cdots & x_{s-1} & x_{s} & x_{s+1} & \cdots & x_{k} \\
r_{0} & \cdots & r_{s-1} & r_{s}-1 & r_{s+1} & \cdots & r_{k}
\end{array}\right)\right. \\
\left.\quad-B_{m-1}\left(x \mid \begin{array}{lllllll}
x_{0} & \cdots & x_{t-1} & x_{t} & x_{t+1} & \cdots & x_{k} \\
r_{0} & \cdots & r_{l-1} & r_{l}-1 & r_{t+1} & \cdots & r_{k}
\end{array}\right)\right)
\end{array}\right\}
$$

Specializing once more to the case $s=k$ and $t=0$ yields the well-known standard recursion formula for $\frac{d t}{d x^{2}} B_{m}$, due to C. deBoor.

Of course our remark in section 2 about possible generalizations of deBoor type algorithms also applies here, i.e. in the case of computing a B-spline curves' derivatives.

Combining equations (2.10) and (3.8), one can now easily prove the following interesting relation between a $\mathrm{B}$-spline and its first derivative; it generalizes a result of G. Meinardus (see [Meinardus [1984]), which is recovered by setting $s=k$ in (3.9). 
Theorem 3.4: For all $s \in\{0, \ldots, k\}$ and $x \in \mathbb{R}$, we have the relation

$$
\begin{aligned}
B_{m}\left(x \mid \begin{array}{llll}
x_{0} & \cdots & \cdots & x_{k} \\
r_{0} & \cdots & \cdots & r_{k}
\end{array}\right)- & \frac{m}{m-1} \cdot B_{m-1}\left(x \mid \begin{array}{lllllll}
x_{0} & \cdots & x_{s-1} & x_{s} & x_{s+1} & \cdots & x_{k} \\
r_{0} & \cdots & r_{s-1} & r_{s}-1 & r_{s+1} & \cdots & r_{k}
\end{array}\right) \\
& =\frac{x-x_{s}}{m-1} \cdot \frac{d}{d x} B_{m}\left(\begin{array}{lllll}
x_{0} & \cdots & \cdots & x_{k} \\
r_{0} & \cdots & \cdots & r_{k}
\end{array}\right) .
\end{aligned}
$$

Proof. Eliminating the term

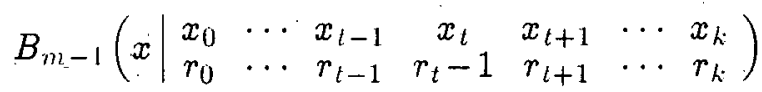

from both equations (2.10) and (3.8), (3.9) is easily established.

We are coming now to the non-trivial problem of computing the partial derivative of a B-spline with respect to the knots $x_{\mu}$. For simplicity of the exposition we restrict ourselves to the case $n=1$ (first derivative); the transfer to higher order derivatives is easily done with similar methods as, for example, in Theorem 3.1 .

Theorem 3.5: Let there be given real numbers $\lambda_{\nu}, \nu=0, \ldots, k$, such that

$$
\sum_{\nu=0}^{k} \lambda_{\nu}=0 \quad \text { and } \quad \sum_{\nu=0}^{k} \lambda_{\nu} x_{\nu}=-1 .
$$

Furthermore pick some index $\mu \in\{0, \ldots, k\}$, and set

$$
\varrho_{\nu}:= \begin{cases}r_{\nu}, & \text { for } \nu=0, \ldots, k, \nu \neq \mu, \\ r_{\nu}+1, & \text { for } \nu=\mu .\end{cases}
$$

Then the following recursion formula for the partial derivative $\frac{\partial}{\partial x_{\mu}} B_{m}$ holds:

$$
\begin{aligned}
& \frac{\partial}{\partial x_{\mu}} B_{m}\left(x \mid \begin{array}{cccc}
x_{0} & \cdots & \cdots & x_{k} \\
r_{0} & \cdots & \cdots & r_{k}
\end{array}\right)= \\
& =r_{\mu} \cdot \sum_{\nu=0}^{k} \lambda_{\nu} \cdot B_{m}\left(x \mid \begin{array}{ccccccc}
x_{0} & \cdots & x_{\nu-1} & x_{\nu} & x_{\nu+1} & \cdots & x_{k} \\
\varrho_{0} & \cdots & \varrho_{\nu-1} & \varrho_{\nu}-1 & \varrho_{\nu+1} & \cdots & \varrho_{k}
\end{array}\right) .
\end{aligned}
$$

Proof. Again we use the contour integral representation (1.2) and find

$$
\begin{aligned}
& r_{\mu} \cdot \sum_{\nu=0}^{k} \lambda_{\nu} \cdot B_{m}\left(x \mid \begin{array}{ccccccc}
x_{0} & \cdots & x_{\nu-1} & x_{\nu} & x_{\nu+1}^{\prime} & \cdots & x_{k} \\
\varrho_{0} & \cdots & \varrho_{\nu-1} & \varrho_{\nu}-1 & \varrho_{\nu+1} & \cdots & \varrho_{k}
\end{array}\right) \\
& =\frac{m \cdot r_{\mu}}{2 \pi i} \int_{C} \frac{\sum_{\nu=0}^{k} \lambda_{\nu}\left(z-x_{\nu}\right) \cdot(z-x)^{m-1} d z}{\left(z-x_{0}\right)^{e_{0}}\left(z-x_{1}\right)^{e_{1}} \cdots\left(z-x_{k}\right)^{e_{k}}} \\
& =\frac{m \cdot r_{\mu}}{2 \pi i} \int_{(} \frac{(z-x)^{m-1} d z}{\left(z-x_{1}\right)^{r_{0}}\left(z-x_{1}\right)^{r_{1}} \cdots\left(z-x_{k}\right)^{r_{k}} \cdot\left(z-x_{\mu^{\prime}}\right)} \\
& =\frac{\partial}{\partial x_{\mu}} B_{m}\left(x \mid \begin{array}{cccc}
x_{0} & \cdots & \cdots & x_{k} \\
r_{0} & \cdots & \cdots & r_{k}
\end{array}\right) \text {. }
\end{aligned}
$$


An alternative proof of Theorem 3.5 can be given based on Theorem 3.1 , using the relation

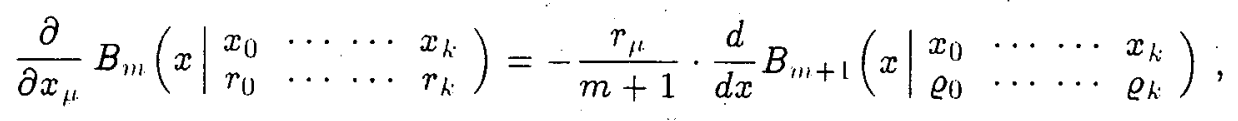

which was proved in [Meinardus\&Walz 1993].

\section{Knot Insertion}

One of the fundamental algorithms for the insertion of knots into B-spline curves was presented by W. Boehm in [Boehm 1980] (cf. also [Farin 1988]). The essential formula of that paper reads, in our notation,

$$
\left.\begin{array}{r}
B_{m}\left(x \mid \begin{array}{llll}
x_{1} & \cdots & \cdots & x_{k} \\
r_{1} & \cdots & \cdots & r_{k}
\end{array}\right)= \\
=\frac{\xi-x_{0}}{x_{k}-x_{0}} \cdot B_{m}\left(\begin{array}{lllllll}
x_{0} & \cdots & x_{j} & \xi & x_{j+1} & \cdots & x_{k} \\
r_{0} & \cdots & r_{j} & 1 & r_{j+1} & \cdots & r_{k}-1
\end{array}\right) \\
\quad+\frac{x_{k}-\xi}{x_{k}-x_{0}} \cdot B_{m}\left(\begin{array}{llllllll}
x & x_{0} & \cdots & x_{j} & \xi & x_{j+1} & \cdots & x_{k} \\
r_{0}-1 & \cdots & r_{j} & 1 & r_{j+1} & \cdots & r_{k}
\end{array}\right) ;
\end{array}\right\}
$$

it connects a B-spline over the old knot sequence with two B-splines over the new one, which is refined by adding the new knot $\xi$.

Using our approach, based on the contour integral formula (1.2), we can now generalize this formula in two ways:

1. We allow an $n$-fold knot to be inserted, and

2. Instead of $x_{k}$ and $x_{0}$, arbitrary knots can be dropped in the B-splines on the right hand side of (4.1).

The generalized knot insertion formula reads as follows:

Theorem 4.1: Let there be given some point $\xi \in\left[x_{0}, x_{k}\right]$, say $x_{j}<\xi<x_{j+1}$. With some natural number $n, n \leq r_{\nu}$ for $\nu=0, \ldots, k$, let there be given real numbers $\lambda_{\nu}, \nu=0, \ldots, k$, satisfying

$$
\sum_{\nu=0}^{k} \lambda_{\nu} x_{\nu}^{\mu \prime}=\xi^{\prime \prime}, \quad \text { for } \mu=0, \ldots, n
$$

Then we have the relation

$$
\begin{aligned}
B_{m}\left(x \mid \begin{array}{cccc}
x_{0} & \cdots & \cdots & x_{k} \\
r_{0} & \cdots & \cdots & r_{k}
\end{array}\right)= \\
\quad=\sum_{\nu=0}^{k} \lambda_{\nu} \cdot B_{m}\left(x \mid \begin{array}{lllllllll}
x_{0} & \cdots & x_{\nu} & \cdots & x_{j} & \xi & x_{j+1} & \cdots & x_{k} \\
r_{0} & \cdots & r_{\nu}-n & \cdots & r_{j} & n & r_{j+1} & \cdots & r_{k}
\end{array}\right) .
\end{aligned}
$$


Sketch of proof. The proof of Theorem 4.1 follows the same idea as that of Theorem 2.1, the essential formula which relates the integrands on both sides of (4.3) is given by

$$
\begin{aligned}
& \frac{(z-x)^{\prime \prime \prime-1} d z}{\left(z-x_{0}\right)^{r_{0}} \cdots\left(z-x_{k}\right)^{r_{k}}}=\frac{\sum_{\nu=0}^{k_{k}} \lambda_{\nu}\left(z-x_{\nu}\right)^{\prime \prime} \cdot(z-x)^{\prime \prime \prime-1} d z}{\left(z-x_{0}\right)^{r_{0}} \cdots\left(z-x_{k}\right)^{r_{k}} \cdot(z-\xi)^{\prime \prime}} \\
& =\sum_{\nu=0}^{k} \lambda_{\mu} \frac{(z-x)^{m-1} d z}{\left(z-x_{0}\right)^{\prime v} \cdots\left(z-x_{1}\right)^{n^{\prime \prime}-11} \cdots\left(z-x_{k}\right)^{\prime k} \cdot(z-\xi)^{\prime \prime}} \text {, }
\end{aligned}
$$

by means of (4.2).

Remarks. 1. It is not necessary that the new knot $\xi$ lies in the interval $\left[x_{11} ; x_{k}\right]$. This was only assumed for notational reasons, but one could choose any real number $\xi$ instead. 2. For fixed $x=\xi$, condition (4.2) coincides with (2.1); this is a new proof of the wellknown fact that the knot insertion algorithm can be viewed as the first step of deBoor's algorithm.

\section{Final Remarks}

The present paper is on B-splines and some of their properties, but what we were in fact talking about was "something which has a contour integral representation of the type given in (1.2)". Now, such contour integrals are by far not restricted to the representation of B-splines, but can be used to represent rather general recursion schemes, see [Brezinski\&Walz 1991]. Only as an example we remark that the (classical) divided difference $\Delta$ can be written as

$$
\Delta\left(x_{0}, \ldots, x_{m} ; \varphi\right)=\frac{1}{2 \pi i} \int_{\mathbb{H}} \frac{\varphi(z) d z}{\left(z-x_{0}\right)\left(z-x_{1}\right) \cdots\left(z-x_{k}\right)}
$$

$\varphi$ being analytic inside the simply closed contour $W$. From (5.1) it can be-seen that most of the present paper's assertions apply also to the divided difference case, thus recovering some of the results in [Mühlbach 1973, 1978] on classical and generalized divided differences.

Similarly there is no reason to be seen why our results should be restricted to polynomial splines. Since there exist contour integral representations similar to that in (1.2) for several types of non-polynomial B-splines (see e.g. [Walz 1989, 1993]), it is most likely that we can find general recursions and knot insertion methods for non-polynomial (e.g. trigonometric or exponential) spline curves as well. This will be investigated in a forthcoming paper. 


\section{References}

Boehm, W. (1980), Inserting New Knots into B-Spline Curves, Computer-Aided Design $12,199-201$.

de Boor, C. (1972), On Calculating with B-Splines, J. Approx. Theory 6, $50-62$.

de Boor, C. (1978), A Practical Guide to Splines, Springer, New York.

Brezinski, C. \& G. Walz (1991), Sequences of Transformations and Triangular Recursion Schemes, with Applications in Numerical Analysis, J. Comp. Appl. Math. 34, $361-383$.

Cox, M. G. (1972), The Numerical Evaluation of B-Splines, Journ. Inst. Math. Appl. 10, $134-149$.

Dahmen, W. (1980), On Multivariate B-Splines, SIAM J. Numer. Anal. 17, 179 - 191.

Farin, G. E. (1988), Curves and Surfaces for Computer Aided Geometric Design, Academic Press, San Diego.

Hoschek, J. \& D. Lasser (1989), Grundlagen der geometrischen Datenverarbeitung, Teubner-Verlag, Stuttgart.

Meinardus, G. (1974), Bemerkungen zur. Theorie der B-Splines, In: Böhmer, Meinardus, Schempp (eds.), Spline-Funktionen, Bibliographisches Institut, Mannheim/Zürich, 165 175.

Meinardus, G. (1984); Introduction to Spline Functions, Quaderno Matematico 95, University of Triest.

Meinardus, G. \& G.Walz (1992), More Results on B-Splines via Recurrence Relations, Math. Manuskripte 144, Universität Mannheim.

Meinardus; G. \& G.Walz (1993), On the Chebyshev Norm of Polynomial B-Splines, Submitted for publication.

Micchelli, C.A. (1979), On a Numerically Efficient Method for Computing Multivariate B-Splines, In: Schempp, Zeller (eds.), Multivariate Approximation Theory (ISNM 51), Birkhäuser, Basel, 211 - 248.

Mühlbach, G. (1973), A Recurrence Formula for Generalized Divided Differences and Some Applications, J.Approx. Theory 9, 165 - 172.

Mühlbach, G. (1978), The General Neville-Aitken-Algorithm and Some Applications, Numer. Math. 31, 97 - 110.

Neamtu, M. (1991), A Contribution to the Theory and Practice of Multivariate Splines, Thesis, University of Twente, Enschede.

Nürnberger, G. (1989), Approximation by Spline Functions, Springer, Berlin/Heidelberg/New York.

Schumaker, L.L. (1981), Spline Functions, Basic Theory, Wiley-Interscience, New York. 
Walz, G. (1989), Remarks on Exponential Splines, in Particular a Contour Integral Represeritation of Exponential B-Splines, Math. Methods Appl. Sci. 11, $821-827$.

Walz, G. (1993), Generalized Divided Differences, with Applications to Generalized BSplines, Calcolo, in Print.

Priv.-Doz.Dr. Guido Walz

Fakultät für Mathematik und Informatik

Universität Mannheim

D-W-6800 Mannheim 1

Germany 